\title{
Flight-Test Validation of a Takeoff Performance Uncertainty Model
}

\author{
András Sóbester* \\ University of Southampton, Southampton, Hampshire, England SO16 7QF, United Kingdom \\ https://doi.org/10.2514/1.C036180
}

\begin{abstract}
A Monte Carlo model designed for fixed-wing aircraft takeoff performance uncertainty quantification is benchmarked. The uses of an efficient takeoff simulator of this type range from rapid design variable and constraint sensitivity studies and large-scale conceptual level analyses to operational performance planning and real-time anomaly detection. The accuracy of the model is assessed against high-resolution flight-test data obtained through a campaign consisting of eight takeoffs flown with a specially instrumented commuter category transport aircraft: a BAe Jetstream Series 3100 twin turboprop. On all but one of the takeoffs, a close agreement is seen in terms of the takeoff distance, as predicted vs as observed, at the point of passing a $35 \mathrm{ft}$ screen height; for the outlier, evidence of a sudden change in wind speed is presented as the probable cause of the discrepancy. Such studies are subject to many other sources of error and uncertainty, which are inherent in both flight-test data analysis and simulation, stemming from the highly dynamic and complex nature of this phase of the flight. The analysis presented also proposes to be a template for dealing with these issues, in a way that is applicable to other benchmarking studies.
\end{abstract}

\section{Nomenclature}

$b \quad=$ wingspan

$C_{D}^{\mathrm{TO}}=$ airframe drag coefficient in takeoff configuration

$C_{D 0}^{\mathrm{TO}}=$ airframe zero-lift drag coefficient in takeoff configuration

$C_{L} \quad=$ airframe lift coefficient

$C_{L \max }^{\mathrm{TO}}=$ maximum lift coefficient in takeoff configuration

$C_{p}$

$C_{t}$

propeller power coefficient

propeller thrust coefficient

total aerodynamic drag vector

$\begin{array}{ll}D_{p} & =\text { propeller diameter } \\ \boldsymbol{F}_{\mathrm{fr}} & =\text { tire rolling resistance vector }\end{array}$

$f_{x}, f_{z}=x$ and $z$ components of the resultant of the forces acting on aircraft

$\boldsymbol{G}=$ weight

$h=$ average height of the wing above ground

$I_{2}=2 \times 2$ identity matrix

$K=$ induced drag coefficient

$\boldsymbol{L}=$ lift vector

$m \quad=\quad$ takeoff mass

$n \quad=\quad$ engine speed

$Q \quad=$ propeller torque
$=$

$=$ runway reaction forc

total thrust vector

engine thrust estimate

coordinate axis attached to runway

coordinate axis perpendicular to runway

body angle of attack

ambient air density

ground effect factor

\section{Introduction}

$\mathbf{T}$ AKEOFF performance drives many design decisions in the conceptual phase; in fact, it even tends to feature prominently in the earliest discussions with the customer around the formulation of the design brief. Tight regulation [e.g., 14 Code of Federal Regulations (CFR) 25.105] and the financial implications of either missing airport (or aircraft carrier) compatibility targets, or paying

Received 27 August 2020; revision received 19 January 2021; accepted for publication 20 June 2021; published online 6 August 2021. Copyright $@ 2021$ by the authors. Published by the American Institute of Aeronautics and Astronautics, Inc., with permission. All requests for copying and permission to reprint should be submitted to CCC at www.copyright.com; employ the eISSN 1533-3868 to initiate your request. See also AIAA Rights and Permissions www.aiaa.org/randp

*Associate Professor, Department of Aeronautics and Astronautics. Senior Member AIAA. cruise efficiency penalties for a propulsion system oversized by the takeoff constraints, focus minds on field performance from the outset. Although this is not a new phenomenon, the current push toward operations from heavily restricted, complex environments (urban mobility) is likely to further increase the demand for a sophisticated, probabilistic understanding of the field performance uncertainties involved, from the earliest stages of the design process.

Takeoff is at the confluence of multiple aircraft design and performance analysis challenges, which, together, conspire to create complex tradeoffs that neither accept elegant, closed-form optima, nor do they ever yield clean, deterministic conclusions. The engineer must thus seek to acquire a probabilistic understanding of what the design brief is likely to cost, which of its entries are the priciest, which design variables drive the constraint boundaries, and where must uncertainty margins be narrowed for smaller variances on the forecast performance. Such variances are likely to exceed those associated with climb and cruise, as more factors are involved: landing gear, runway shape and surface, wind variability, high-lift system aerodynamics, ground effect, propulsion system transients, piloting technique, etc., all propagating through to the distributions of the all-important takeoff distances (all engines operating, critical engine failure at decision speed, etc.).

The starting point of this paper is a probabilistic simulation framework constructed around a fast, efficient numerical solver of the point mass equations of motion that govern the takeoff run. Let us examine the suitability of such a model for generating probabilistic takeoff performance estimates via a Monte Carlo approach. Here, "suitable" stands for 1) computationally inexpensive, to the extent of plausibly serving as a real-time decision support tool running on a laptop in a design meeting or in a preliminary discussion with a customer; and 2) of an accuracy convincingly benchmarked against the real world. Although testing definition 1 requires little more than a simple convergence analysis and a stopwatch, definition 2 presents significant challenges due to the inherent errors in any observed flight-test data, as well as due to the aforementioned complexities of field performance having to be dealt with both in terms of the benchmark and the prediction model.

We tackle the benchmarking via a series of flight tests conducted with G-NFLA, a BAe Jetstream Series 3100 aircraft. It serves the purpose exceptionally well, thanks to its high-quality, rigorously calibrated instrumentation. Moreover, its operator [the U.K. National Flying Laboratory Centre (NFLC)] has amassed an almost peerless wealth of performance data on the aircraft over its long years of service, providing not only estimates of its characteristics, but also confidence intervals around these, which are essential for the complex benchmarking task at hand.

Although design applications motivated this work, a fast, probabilistic field performance model has operational applications too, for example, in real-time anomaly monitoring. Specifically, it may be an 
effective means of the early automated rejection of takeoffs (e.g., in the case of remotely piloted/autonomous aircraft), where a continuation condition may be defined in terms of percentile targets set by the prediction model (and, given sufficient onboard compute power, reevaluated several times during the ground roll). The reader interested in this type of application may wish to consult, for example, the work of Zollitsch et al. [1]. Here, we shall mainly adopt a design perspective.

\section{Uncertainty in Field Performance Prediction}

The design brief and its translation into a design concept commit the aircraft manufacturer to choices with "long tails" spanning the preliminary and detail design phases of the development, as well as the rest of the life cycle of the product. The weight of this earliest phase of the development process is compounded by the uncertainties arising from the lack of a geometry (beyond, often, a topological sketch), and thus the absence of detailed simulations and tests; in fact, most design variables and performance estimates carry large uncertainty margins at this stage. This is compounded by the uncertainties of the environment and the geography of the future operators of the aircraft.

Of course, it is possible to size early concepts for limit cases (e.g., in the takeoff performance context, using a "one-percentile low-density" MIL-HDBK-310 [2] atmosphere at KDEN), but this yields far less insight into sensitivities than a full propagation of distributions through the design. [Denver International Airport (elevation $5434 \mathrm{ft}$ ) is a classic design point for transport aircraft takeoff and first/second segment climb performance.] Moreover, it offers no possibility of calculating the integrals of key quantities, such as one may need for an early estimate of the environmental impact (see, for instance, the work of Allaire et al. [3] on estimating the distributions of noise and emissions metrics for specific locations).

In a pair of papers on integrating aerodynamic uncertainty into aircraft maneuvers, Wendorff et al. [4,5] make the point that such techniques are powerful tools in de-risking certification, which is where an approach that yields probabilities of meeting certain requirements becomes far more valuable than a "best estimate" or "best estimate of worst case" approach, flagging potential issues as early as the conceptual design phase. Uncertainty quantification in the early stages of the design process is also the foundation of robust design approaches; see, for example, the conceptual-design level Monte Carlo analysis of aircraft performance metrics reported by $\mathrm{Ng}$ and Willcox [6], where they tackle the optimization of a NASA N+3 subsonic transport under uncertainty.

The rapidly growing general literature on uncertainty quantification and robust design is beyond the scope of this paper, but let us consider uncertainty briefly from the perspective of takeoff performance.

One of the most recent in-depth analyses of the topic is due to Takahashi et al., who examined the impact of pilot technique [7] and aeropropulsive factors [8]. As an instance of the former, they highlight the impact of pitch rate in the transition from ground roll to flight (rotation), noting the lack of standardization and a lack of training received by crews in terms of the impact of deviations from the optimum (erring either on the side of under-rotation, resulting in increased takeoff distances, or over-rotation, resulting in the same outcome due to excessive alpha or tail strike). Takahashi et al. model the takeoff of an A320-class twin jet and estimate an impact of slower-than-prescribed rotation of up to $30 \%$ on takeoff field length. Another remarkable result of their simulations is that a 1 deg reduction in the maximum ground angle of attack (AoA; from 18 to $17 \mathrm{deg}$, such as one might expect when implementing a fuselage stretch) can increase certified distances by more than 5\% (assuming that the wing remains unchanged) []ㅡ.

The operational impact of such uncertainties and our inability to quantify them accurately is that, to quote Bays and Halpin [9], the "conservatism inherent in traditional manuals produces an undue penalty in airfield performance." An example is the common feature found even in computational field performance planning models that calculations are based on half of the actual headwind component, and a tailwind component is typically multiplied by a safety factor of 1.5 [10], both designed to cover the uncertain impact of some of the factors mentioned previously. (Typically, several other inputs are penalized in similar ways.) Further, "there is no industry standard," Bays and Halpin go on to write, "for establishing the appropriate level of conservatism relative to the physics-based models upon which the traditional manuals are based." The end result of all of the above is poorly quantified project risk (in a conceptual design setting) and unnecessarily conservative airfield constraints (in the context of the operations of existing aircraft).

Interest in a better understanding of field performance uncertainty is likely to increase in the coming years, carried by the rising tide of the quest for the most effective urban mobility solution. Discussions around this topic are often dominated by multirotor vertical takeoff and landing (VTOL) concepts, but there is a school of thought that advocates fixed-wing solutions with distributed electric propulsion as a faster, more viable route to effective urban mobility [11], which can be built on an existing airworthiness framework (such as 14 CFR Part 23) and may suffer less from the noise issues and technological risks of VTOL ideas. Such concepts hinge, however, on their feasibility in tight, cluttered, complex environments, often featuring "tabletop" runways on the roofs of buildings, and complicated failure and abort scenarios. They will thus, perhaps even more than conventional applications, require a validated, probabilistic takeoff performance framework, and it is such a tool that we propose next.

\section{Numerical Model for Rapid Takeoff Simulation}

\section{A. Solving the Equations of Motion}

In the context of conceptual design level takeoff performance, modeling the customary approach is to solve the equations of motion in the time $t$ domain, with the aircraft represented by a point mass $m$, moving in the $(x, z)$ plane, where $x$ is a horizontal coordinate axis with the same azimuth as the runway centerline (positive in the takeoff run direction) and $z$ is vertical, positive upward (see, e.g., Blake [12] and Takahashi [13]). We adopt the same starting point here. Designating the origins of both axes as the point where the aircraft begins its ground roll from rest, we assume the motion of the aircraft to be described by the system of ordinary differential equations:

$$
\left[\begin{array}{l}
\ddot{x}(t) \\
\ddot{z}(t)
\end{array}\right]=\frac{1}{m} \boldsymbol{I}_{2}\left[\begin{array}{l}
f_{x}(t, \dot{x}(t), \ldots) \\
f_{z}(t, \dot{z}(t), \ldots)
\end{array}\right]
$$

and we consider the model of the takeoff as an initial value problem, where

$$
x(0)=0, \dot{x}(0)=0, \ddot{x}(0)=0, z(0)=0, \dot{z}(0)=0, \ddot{z}(0)=0
$$

and

$$
\left[\begin{array}{c}
f_{x}(t, \dot{x}(t), \ldots) \\
f_{z}(t, \dot{z}(t), \ldots)
\end{array}\right]=\boldsymbol{T}+\boldsymbol{D}+\boldsymbol{L}+\boldsymbol{R}+\boldsymbol{G}+\boldsymbol{F}_{\mathrm{fr}}
$$

The right-hand side represents the vector sum (in a plane aligned with the runway centerline and perpendicular to its surface) of the forces acting on the aircraft: the thrust $\boldsymbol{T}$, the total drag $\boldsymbol{D}$, the net lift $\boldsymbol{L}$, the reaction force acting on the tires during the ground roll $\boldsymbol{R}$, the takeoff weight $\boldsymbol{G}$, and the rolling resistance of the tires $\boldsymbol{F}_{\mathrm{fr}}=\mu \boldsymbol{R}$. Closed-form analytical solutions are rendered impossible by the complex relationships between these terms. For example, $\boldsymbol{T}$ is typically a function of $\dot{x}(t)$, but is also a function of throttle setting, which, in turn, can be a function of $t$ [or even $x(t)$ ] itself, to allow the modeling of the spool-up transients of the engine, as well as various engine failure profiles (e.g., "throttle chop").

A peculiarity of the takeoff modeling problem is that its time domain alternates long periods of limited variation in the derivatives (most of the ground roll and the climb out) with relatively challenging transients (spool up and rotation). Although constant step-size Runge/Kutta-type schemes (e.g., classical Euler integration) are the simplest to implement (and thus popular), their step size will be set by the requirements associated with the transients, and will therefore be 


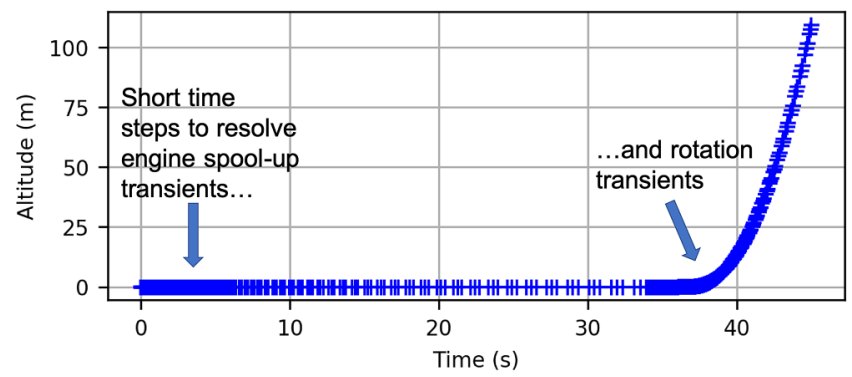

Fig. 1 Bogacki-Shampine time steps marked by "+" symbols on the altitude profile of a simulated takeoff run.

wasteful elsewhere. Our desire to integrate the simulation into a Monte Carlo framework puts pressure on the computational efficiency of the solution, and so here we opted for an adaptive step-size approach, specifically a three-stage third-order Runge-Kutta method based on the Bogacki-Shampine formula [14] (as implemented in [15]), which, throughout the experiments described here, has proved both fast and robustly stable with a residual threshold of $10^{-7}$. Figure 1 illustrates the time-domain resolution variation during a typical takeoff run simulated via adaptive Runge-Kutta time stepping.

\section{B. Uncertainty Quantification}

The popularity of the forward-propagation Monte Carlo method for this type of application is largely the result of its nonintrusive nature; the governing equations can be solved in the same way as one would solve the deterministic problem, and it can be applied to black-box analysis codes too. It also handles large numbers of factors in problems featuring complex, multidisciplinary interactions; see, for example, the work of Siva et al. [16] on dealing with the interactions between structures and aerodynamics in helicopter performance analysis, or recent tilt-rotor analysis work at NASA Ames Research Center (Khurana et al. [17]). It is also naturally concurrent, allowing linear wall time control by varying the numbers of processors deployed on the problem.
Another strength of the Monte Carlo approach in the aircraft design context is that it also works well when the uncertainties of the problem are captured through a mix of analytically postulated probability density models (e.g., Gaussian) and real-world ensembles of observations, such as environmental data sets; see, as an illustration, our earlier work on incorporating atmospheric profile ensembles into a forecast of balloon landing site distributions [18] via a Monte Carlo calculation. In the context of takeoff performance modeling, other observational data sets (beyond weather) might include pilot input profiles collected in a flight simulator (see, e.g., the work of Wood et al. [19] observing variations in takeoff technique in a CRJ-200 flight simulator) or engine power loss profiles (e.g., collected during simulated failures on a test stand).

Figure 2 is a sketch of our proposed architecture, highlighting the principal data flow paths. Each component with a blue background is a probabilistic data source. They supply weather data, contingencies, aerodynamic parameters, and pilot inputs, as driven by the outer Monte Carlo loop. In the validation study described in Sec. IV, the aerodynamics module will constitute the principal source of uncertainty, but the framework is capable of deploying all (or any subset of) these sources for a design study.

The network of information flow arrows highlights one of the implementation challenges of such a simulator, namely, that the solver operates in the time domain, but the various terms on the right-hand side of the equations of motion (3) are functions of velocity and other parameters; moreover, the adaptive nature of the chosen Runge-Kutta solver breaks the linearity of the time domain. (Refinement iterations require unpredictable returns over already-covered ground, in both time and space.) This necessitates careful, yet computationally efficient bookkeeping to "straighten" the arrow of time to deal with timedependent transients, such as engine spool up and rotation.

A key control lever for any simulation is the size of the Monte Carlo sample; the convergence traces in Fig. $\underline{3}$, generated on the validation scenario described in the next section, offer an insight into the associated simulation time/accuracy tradeoff. Both the sample means and the sample standard deviations require of the order of 1000 simulations before the fluctuations settle down to 1-2 $\mathrm{m}$ in terms of the various landmark distances of the takeoff.

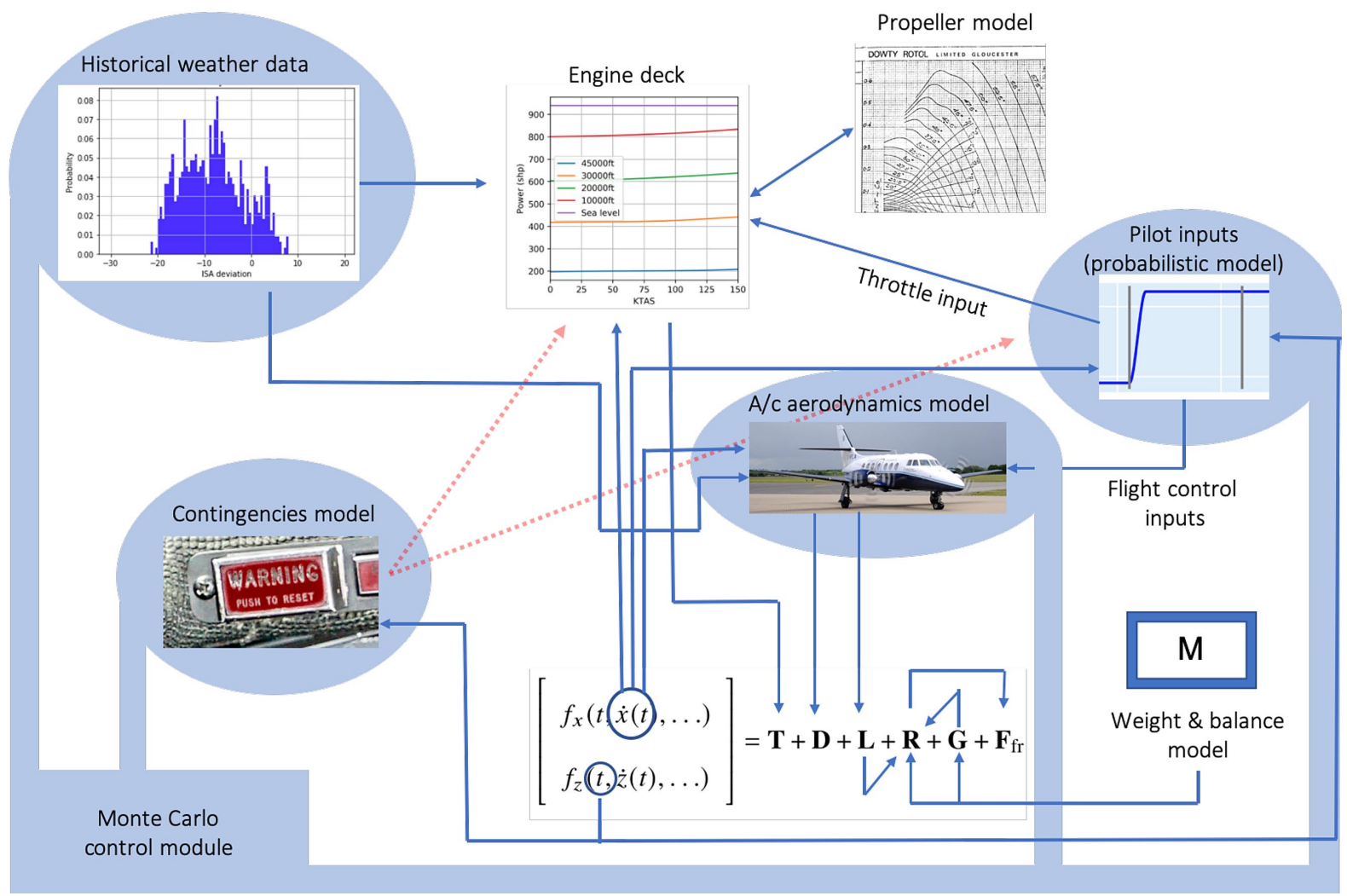

Fig. 2 Probabilistic takeoff simulation framework. 

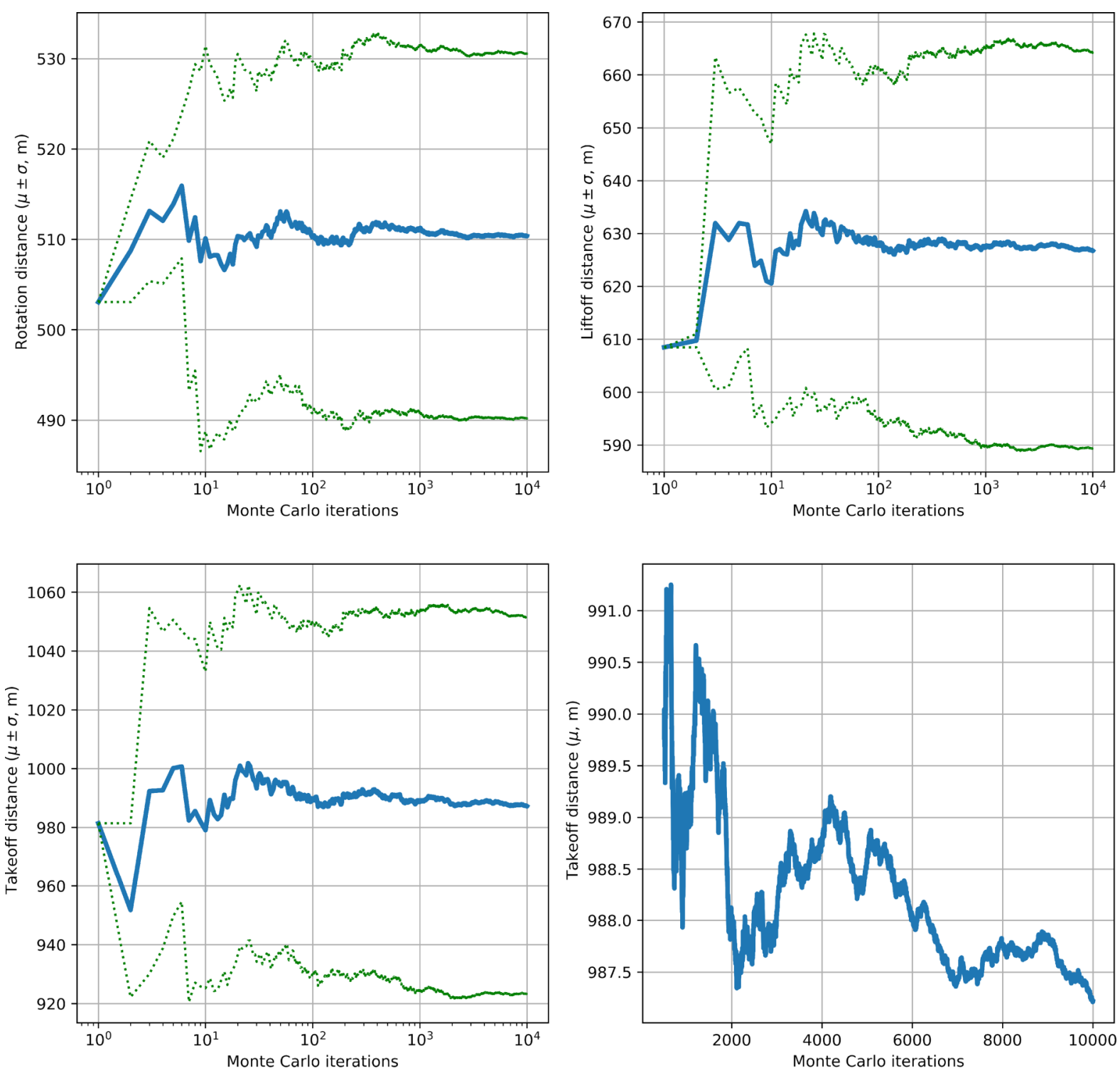

Fig. 3 Monte Carlo convergence study: typical mean (blue) and standard deviation (dotted green) traces.

\section{Validation Flight Tests: Aircraft, Airfield, Conditions, and Limitations}

We have chosen to validate the model using high-resolution takeoff performance data recorded onboard the BAe Jetstream Series 3100 aircraft, registration G-NFLA (see Fig. 4), of the U.K. NFLC. The validation data set used in this paper consists of the variable traces of eight takeoffs, performed by an NFLC crew at Southampton Airport (EGHI) at takeoff weights ranging from 6372 to $6722 \mathrm{~kg}$ (spanning a little under $5 \%$ of the maximum takeoff weight of $7059 \mathrm{~kg}$ of G-NFLA).

\section{A. Test Environment}

All takeoffs were flown from runway 20 [with a takeoff run available (TORA)/accelerate-stop distance available of $1650 \mathrm{~m}$ and a takeoff distance available (TODA) of $1805 \mathrm{~m}$ ]. The lineup point of runway 20 has an elevation of $44 \mathrm{ft}$; the variable atmospheric conditions during the test campaign translated into density altitudes ranging here from -96 to $312 \mathrm{ft}$ through the eight flights. The weather conditions during the flights were variable, ranging from dry and sunny through overcast with light drizzle, light showers, and light rain. Table 1 is a summary of the environmental conditions recorded at the time of each takeoff, as follows:

1) Outside air temperature (OAT) is calculated as the average of the airport METAR (standard format for reporting a summary of the weather observations at and in the vicinity of an aerodrome) observations published just before and just after each takeoff and the mean of the OAT signal recorded by the air data system of the aircraft; rounded to the nearest degree (the exact value was used in the calculations)

2) QFE is the ambient pressure at the airfield level [see the METAR for the QNH (QFE, QNH: altimeter pressure setting for the surface and sea level, respectively)]; expressed in millibars ( $\mathrm{hPa}$ ), rounded to the nearest millibar

3) The density altitude is expressed in feet; rounded to the nearest $\mathrm{ft}$

4) The headwind component on runway 20 is expressed in knots; rounded to the nearest knot

5) Southampton Airport METARs (aerodrome weather information): are issued at $30 \mathrm{~min}$ intervals. The METAR preceding the takeoff and the one immediately following it (shaded in blue) are shown in the table. The time stamp of the latter was nearer to the actual takeoff times; thus, the wind value recorded therein was used in the simulations conducted for validation purposes. Only the weather section of each METAR is shown, with the International Civil Aviation Organization (ICAO) identifier (EGHI) and the date and time block omitted for compactness

Runway 20 has a listed slope of $-0.23 \%$, but this is simply the slope of a straight line connecting its two ends. G-NFLA lifted off on each of our test flights nearer the halfway point of the runway, up to which point the downslope is significantly greater (due to the "V" 


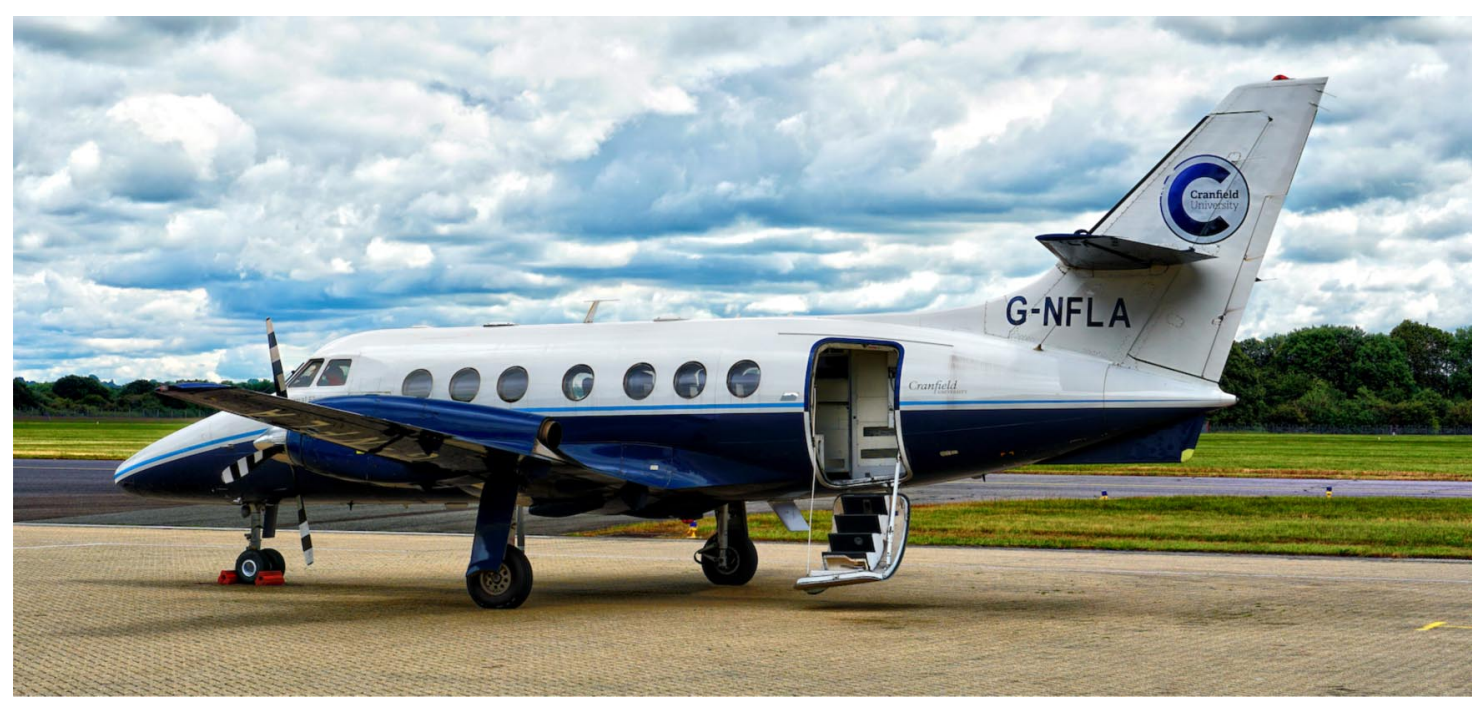

Fig. 4 National Flying Laboratory (Jetstream 31; G-NFLA) being prepared for the test flights (photo by the author).

Table 1 Environmental conditions during the eight validation takeoffs

\begin{tabular}{|c|c|c|c|c|c|}
\hline Flight no. & $\mathrm{OAT},{ }^{\circ} \mathrm{C}$ & QFE, mbar & $\begin{array}{l}\text { Density } \\
\text { altitude, } \mathrm{ft}\end{array}$ & $\begin{array}{l}\text { Headwind } \\
\text { component, } \mathrm{kt}\end{array}$ & $\begin{array}{l}\text { METAR (ICAO code and time block omitted for compactness) last report } \\
\text { before takeoff first report after takeoff (nearest in time, used for wind estimate) }\end{array}$ \\
\hline 1 & 12 & 1004 & -96 & 13 & $\begin{array}{l}20013 \text { KT 170V250 } 6000 \text { RA BKN012 12/08 Q1005 } \\
20013 \text { KT } 7000 \text { RA FEW010 BKN013 12/08 Q1006 }\end{array}$ \\
\hline 2 & 12 & 1005 & -73 & 13 & $\begin{array}{l}20014 \text { KT 170V230 } 7000 \text { RA FEW012 BKN015 11/09 Q1006 } \\
20013 \text { KT 160V230 } 9999 \text {-RA BKN012 12/09 Q1006 }\end{array}$ \\
\hline 3 & 13 & 1005 & 7 & 16 & $\begin{array}{l}21015 \text { KT } 9999 \text {-RA FEW011 BKN014 12/09 Q1006 } \\
21016 \text { KT 170V240 } 9999 \text { VCSH SCT012 BKN016 12/08 Q1006 }\end{array}$ \\
\hline 4 & 13 & 1005 & 47 & 15 & $\begin{array}{l}\text { 21018G30 KT 180V240 9999 -SHRA SCT012 BKN016 12/09 Q1006 } \\
21015 \text { KT 180V240 } 9999 \text {-SHRA SCT014 BKN016 13/09 Q1006 }\end{array}$ \\
\hline 5 & 15 & 1009 & 109 & 14 & $\begin{array}{l}19014 \text { KT 170V240 } 9999 \text { FEW013 BKN025 15/10 Q1010 } \\
20014 \text { KT 170V230 9999 FEW013 BKN025 15/10 Q1010 } \\
\end{array}$ \\
\hline 6 & 15 & 1009 & 149 & 16 & $\begin{array}{l}20015 \text { KT 160V240 } 9999 \text { FEW015 BKN025 15/09 Q1010 } \\
\text { 19016 KT 150V220 } 9999 \text {-DZ FEW016 BKN025 15/09 Q1010 }\end{array}$ \\
\hline 7 & 17 & 1010 & 312 & 17 & $\begin{array}{l}20015 \text { KT 150V240 } 9999 \text { FEW016 BKN025 16/09 Q1011 } \\
\text { 20017G27 KT 170V230 9999 FEW016 SCT025 16/09 Q1011 }\end{array}$ \\
\hline 8 & 16 & 1010 & 233 & 15 & $\begin{array}{l}21015 \text { KT 180V240 } 9999 \text { BKN021 15/08 Q1011 } \\
20015 \text { KT 170V230 } 9999 \text { FEW020 BKN025 15/07 Q1011 }\end{array}$ \\
\hline
\end{tabular}

profile of the runway). We were able to use the inertial altitude recorded by the inertial reference system (IRS) of the aircraft as a means of charting the actual topographical profile of the section of the runway used for the takeoff. We have inserted the slope values thus found (typically of the order of -1 to $-2 \%$, the variation among them being due to the different ground-roll distances) into our simulator, for the validation cases.

\section{B. Propulsion}

The test aircraft is powered by two Garrett AiResearch (now Honeywell) TPE331-10UR-513H turboprop engines. They have a single-shaft architecture, featuring a two-stage centrifugal compressor, a three-stage axial turbine, and an annular combustion chamber. Each of the TPE331s fitted to G-NFLA drives a four-bladed DowtyRotol propeller, designed to operate at a constant speed when the rpm lever in the flight deck is in "FLIGHT" mode (including at takeoff). In this mode, the governor controls the blade angles such that a propeller speed of $1591 \mathrm{rpm}$ is maintained.

The TPE331-10UR series of engines has a thermodynamic (full) rating of $1000 \mathrm{shp}$, flat rated to $940 \mathrm{shp}$ for takeoff $(5 \mathrm{~min}$ of operation; see Fig. 5); the maximum continuous flat power rating is $900 \mathrm{shp}$. Jet propulsion accounts for around 5\% of the total thrust generated by each engine (derived from the equivalent shaft powers

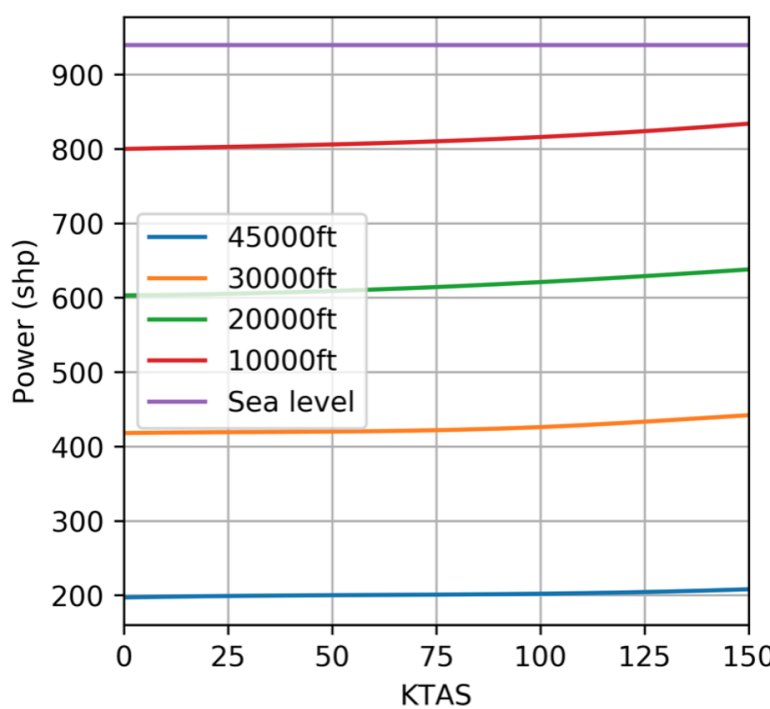

Fig. 5 Uninstalled 5 min maximum power curves of the TPE331-10UR engine (from data presented in the manufacturer's specifications document [21]). 
quoted by the type certificate as 984 and 944 eshp for takeoff and maximum continuous operation, respectively) [20,21]. All these are uninstalled figures, that is, they relate to an engine operating on its own, on a test rig. Typical installation losses, accounting for engine-airframe integration effects, as well as bleed air offtakes and other services provided by the powerplant, are estimated by Kundu [22] at between 8 and $10 \%$ at takeoff; the Monte Carlo simulations performed here as benchmarks for our validation cases assume a normally distributed figure, with Kundu's numbers setting the 95 percentile boundaries.

The operating limits of the engines on Jetstream 31 are guarded by a torque and temperature limiter computer, which limits the fuel flow to the engines to prevent either the maximum torque or maximum exhaust gas temperature (EGT) red line limit of $650^{\circ} \mathrm{C}$ from being exceeded. In case of engine failure (detected as a sudden loss of torque), an automatic power reserve logic adds $19^{\circ} \mathrm{C}$ of "headroom" to the EGT limit of the other engine [23].

For purposes of the validation study at the center of this paper, we have constructed a model of the performance of the engine, with environmental conditions, power lever position, true airspeed (TAS), and torque gauge reading as inputs and thrust as the output. Here is the algorithm that forms the basis of the model. First, the torque gauge reading is converted into an actual torque value using a calibration coefficient obtained experimentally (using the Lebow test procedure) by the NFLC via a specialist calibration facility. This allows the calculation of the power coefficient:

$$
C_{p}=\frac{2 \pi Q}{\rho n^{2} D_{p}^{5}}
$$

where $Q$ is the torque, $\rho$ is the ambient density, $n$ is the engine speed ( $26.5 \mathrm{rev} / \mathrm{s}$ in the FLIGHT mode of the propeller governor (the mode selected at takeoff), and $D_{p}$ is the diameter of the propeller $(2.692 \mathrm{~m})$. The $C_{p}$ value thus computed, along with the advance ratio of the propeller (the ratio of the TAS and the tip speed), is the input to a pair of models supplied by Dowty (the manufacturer of the propeller) that allow the calculation of the blade angle, and finally, the thrust coefficient $C_{t}$, which, along with the original inputs, yields the single engine thrust estimate $T_{e}$ as

$$
T_{e}=C_{t} \rho n^{2} D_{p}^{4}
$$

\section{Airframe}

G-NFLA is an ideal validation case for two reasons. First, the aircraft is equipped with an extensive suite of sensors and a highresolution data logging capability (more on this presently). Second, over its long service, the NFLC was able to amass a robust and detailed understanding of its performance and aerodynamics. The following is a distillation of this wealth of data (based on [24-26]).

Most germane to the study at hand are the key aerodynamic parameters of the airframe in the takeoff configuration (flaps $10 \mathrm{deg}$; undercarriage down). The zero alpha lift coefficient $C_{L 0}^{\mathrm{TO}}$ of the airframe, derived with a 95 percentile confidence interval of \pm 0.008 , is 0.523 , with a slope of $a_{1}^{\mathrm{TO}}=5.8 \mathrm{rad}^{-1}$ on the linear portion $C_{L}^{\mathrm{TO}}=C_{L 0}^{\mathrm{TO}}+$ $a_{1} \alpha_{b}$ of the lift curve defined in terms of the body AOA $\alpha_{b}$. The airframe achieves its maximum lift coefficient $C_{L \max }^{\mathrm{TO}}=1.81$ in the takeoff configuration at $\alpha_{b}=9.7 \mathrm{deg}$ (with $95 \%$ of observations in the \pm 0.5 deg interval).

The zero-lift drag coefficient $C_{D 0}^{\mathrm{TO}}$ of the airframe, derived with a 95 percentile confidence interval of \pm 0.001 , is 0.072 . On the assumption of a parabolic drag polar of the form $C_{D}^{\mathrm{TO}}=C_{D 0}^{\mathrm{TO}}+K \Phi C_{L}^{2}$, the induced drag coefficient $K$ was determined through extensive flight testing as $0.056 \pm 0.001$. (It is safe to assume this being constant through the low-Mach-number range of the aircraft.) The ground effect factor $\Phi$ accounts for the presence of the runway and is estimated as

$$
\Phi=\frac{(16 h / b)^{2}}{1+(16 h / b)^{2}}
$$

where $h / b$ is the ratio of the height of the wing above the ground and the span of the main wing $[27,28]$.

A final note on the takeoff aerodynamics of the Jetstream 31: the "clean" airframe generates 371 counts of zero-lift drag; thus, lowering the landing gear and deploying the flaps to 10 deg effectively double $C_{D 0}$ !

\section{Data Recording and Processing}

G-NFLA is equipped with a Litton LTN-90 IRS, which uses pendulous accelerometers, ring laser gyroscopes, and a GPS unit. A key parameter from the viewpoint of validating our simulation capability was the position (and, implicitly, the ground speed) of the test aircraft. Figure 6 is an illustration of the error margins we were able to place around the GPS latitude data. The arrays of gray disks represent individual latitude readings along a typical takeoff run, and they show that the update rate of the GPS unit is considerably lower than the overall sampling frequency of the reference system. We have constructed sixth-order polynomials to place a lower and upper bound on the latitude signal (blue and green curves, respectively). The colored patches superimposed on an aerial view of the runway represent this uncertainty range mapped onto the tarmac at a series of instants along a typical ground roll (initial position and 5, 10, 15, 20, and $25 \mathrm{~s}$ ). Taking advantage of the fact that the aircraft is known to have followed the centerline of the runway during the ground roll, we have implemented a "snap-to-road" algorithm (commonly used in incar satellite navigation) to compute the longitude at each sample.

Although a highly precise reconstruction of the full kinematics is not necessary for model validation purposes, the analysis outlined previously was important to allow us to identify the end-of-takeoff landmark, the point where the aircraft passed the $35 \mathrm{ft}$ screen height. (We would proceed to extract the same reference point from the simulations too for this key comparison.)

Additionally, the readings from the LTN-90 and the air data unit of the aircraft contributed to our reverse engineering of the "real-life" performance of the engine. As outlined earlier, this involved calculating the thrust, which relies on the readings from the torque-sensing system built into the propeller and a TAS input. The accuracy of the thrust estimation is limited in the first $5 \mathrm{~s}$ or so of the ground roll by the fact that G-NFLA is not equipped with a direct rpm and blade angle sensing capability; thus, our calculations had to be made on the assumption that the propeller speed stayed constant (at the FLIGHT mode target speed of $1591 \mathrm{rpm}$ ) through the initial spool-up phase, with the governor reacting without significant lag to the substantial increase in power setting. For the purposes of the simulation, we approximated the throttle setting (which is also not logged directly by G-NFLA) with a sigmoid function spanning the approximate spoolup time we reverse engineered from the flight-test data (with the aforementioned caveat).

Another issue related to the first few seconds of the ground roll is the measurement of the airspeed. The TAS, in addition to being required by the thrust computation, is also an input of the benchmarking simulations, more specifically, its headwind component. In theory, the difference between the TAS reported by the air data unit of the aircraft and the GPS-derived ground speed should yield the headwind, which could then be fed into the simulation as part of the validation test. In practice, however, this is problematic for two reasons. One is the aforementioned GPS position uncertainty (compounded here by differentiation). The other is that the TAS reading is heavily affected at the start of the rollout by the proximity of the pitot tubes of the Jetstream to the propeller disks; specifically, the TAS signal spikes with the sharp power increase associated with the beginning of the roll. Figure 7 depicts the interplay between these parameters on a typical takeoff run. A key reference point here (black dotted line), which we eventually proceeded to use as the runway component of the wind-speed value in the simulation, is the runway component of the wind recorded in the METARs.

The METARs also indicate gusts and variable wind directions through the campaign (see Table 1), which introduces an additional source of uncertainty, but it can be seen in Fig. 7 that the "TAS minus ground speed" estimates do converge upon the METAR-recorded 


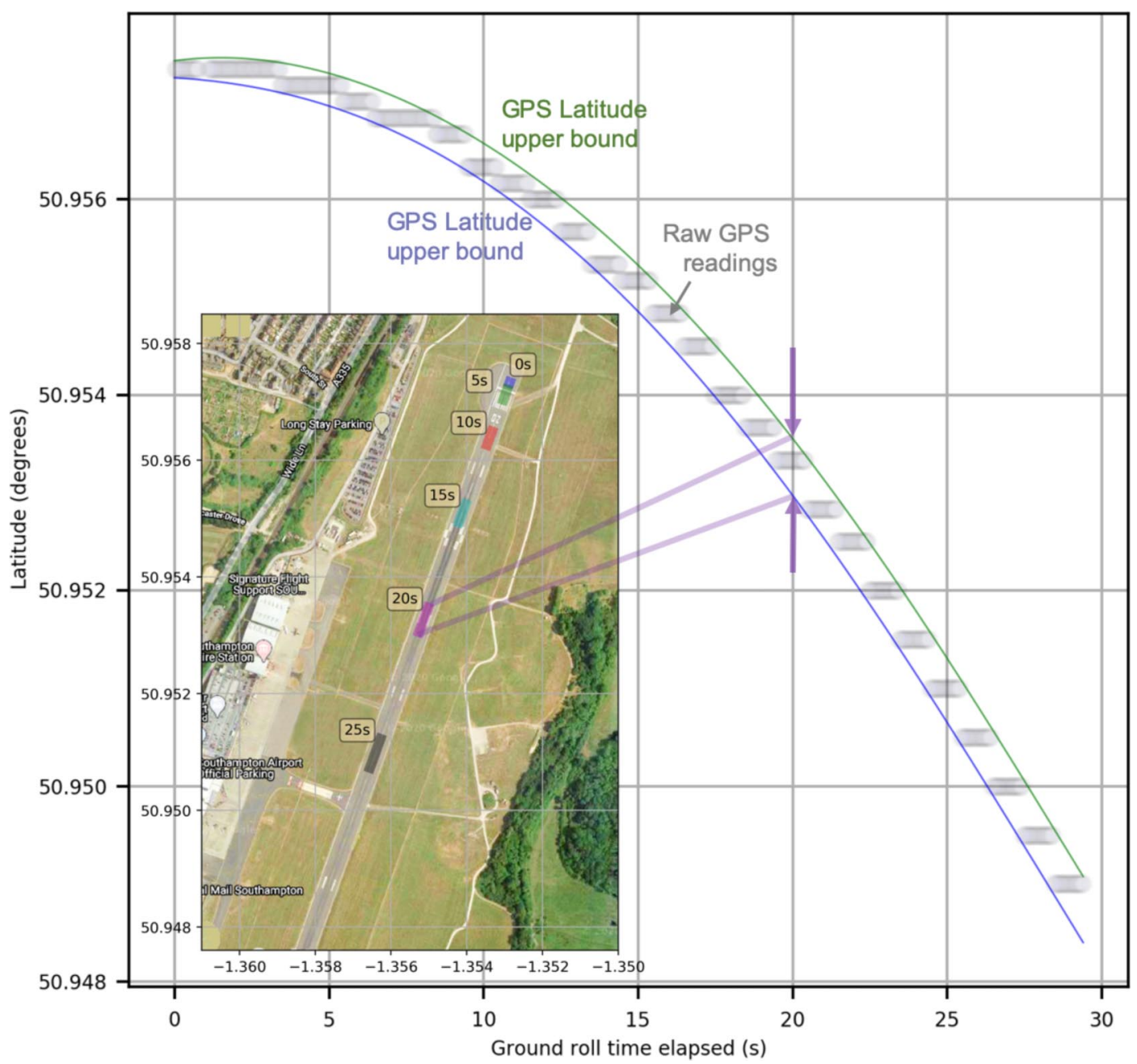

Fig. 6 Upper and lower bounds on the GPS latitude readings mapped onto the runway for a particular moment in time (20 s).

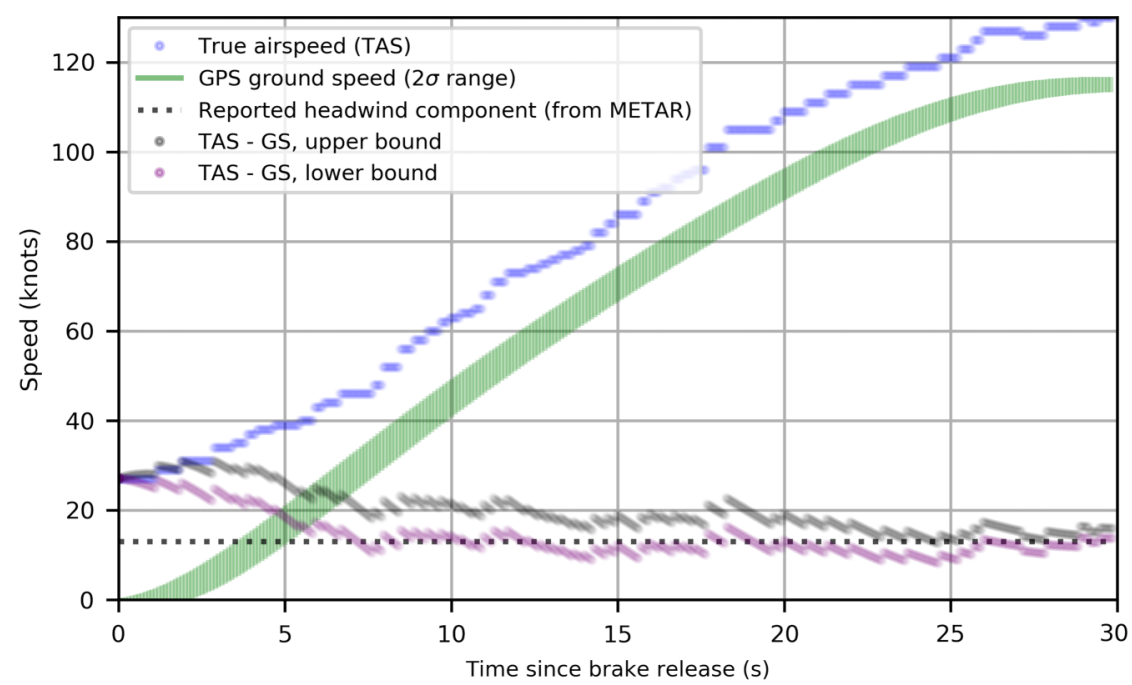

Fig. 7 Headwind component estimates from two sources: aerodrome observations and onboard sensors.

value in the latter part of the takeoff run. We shall return to this aspect of the data analysis during our discussion of the results of the benchmarking study.

Further uncertainties lie in the IRS data in terms of noise in the signal and resolution limitations. Some of the noise is inherent to the physical limitations of the ring laser gyros, whereas others reflect the "untidiness" of the environment (e.g., the response of the landing gear oleos to discontinuities in the pavement, gusts, propulsion system vibrations, and natural unevenness of human pilot inputs). Consider Fig. 8, a plot of $2 \mathrm{~s}$ or so of raw data generated by the LTN90 during one of the takeoffs, showing the beginning of the rotation phase (nose gear lifting off the runway). Some of the air data (AOA) and control surface positions are also shown.

Clearly, rotation occurs around the $22 \mathrm{~s}$ mark, but, as confirmed by the rest of the data from this campaign, it is hard to pin down the exact moment to a precision greater than to within a second or so, due to the 

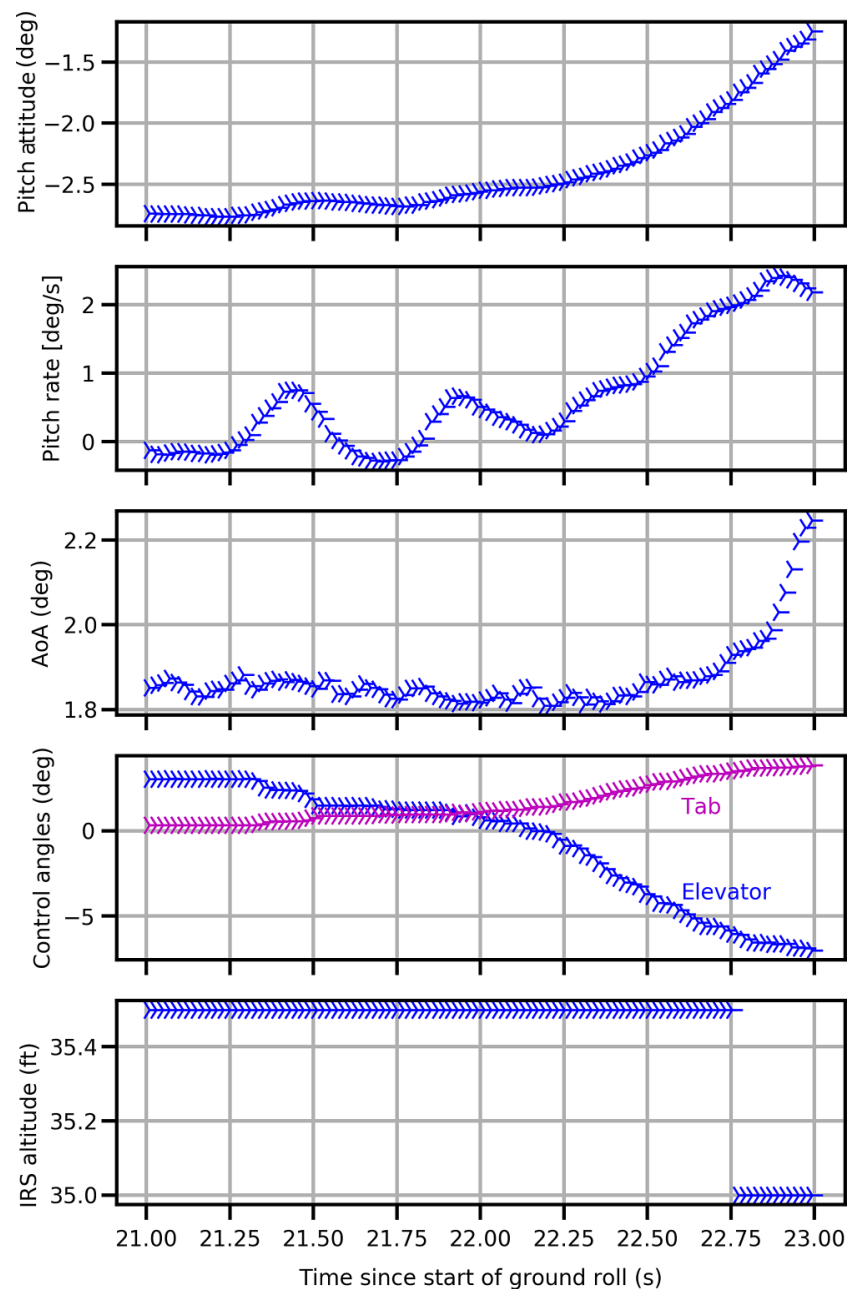

Fig. 8 Two seconds of raw data recorded during one of the takeoffs; rotation occurs approximately between 21.5 and $22.5 \mathrm{~s}$.

various fluctuations and lags in the recorded data. The IRS altitude was, in most of the cases we examined here, perhaps counterintuitively, one of the safest indicators of rotation having definitely occurred, as the IRS unit is mounted aft of the main gear, and therefore, rotation results in a small dip in its altitude; however, this merely gives us an upper bound, as the accelerometer needs approximately one foot of travel before it integrates any motion from the acceleration. All we can conclude from this particular snapshot of the data is that by $22.75 \mathrm{~s}$, the rotation phase will definitely have been in progress. (Also, this dip is somewhat confounded by those caused by unevenness in the runway surface.) We were also able to bracket the other milestones (the start of the ground roll, passing the velocity of minimum control on the ground, liftoff, and completion of takeoff) into a $1 \mathrm{~s}$ window.

\section{E. Piecing It All Together}

The analysis outlined previously has allowed us to identify the salient features of each of the eight test flights. Key elements of the data corresponding to the first of these takeoffs (taken as an example) are depicted in Fig. 9, overlaid on top of a map of Southampton Airport.

Milestones of the takeoff are shown on the right-hand side, each with its own position uncertainty band projected onto the runway (resulting from the uncertainties around time and latitude/longitude, as discussed previously). The runway itself is colored, corresponding to the along-track component of the acceleration of the aircraft, peaking early on at just over $0.25 \mathrm{~g}$, a value fairly typical of transport aircraft operations. The Jetstream takes approximately $17 \mathrm{~s}$ (over half of the total takeoff time) to reach its minimum ground control speed of $90 \mathrm{kt}$ indicated airspeed (KIAS), with the crew initiating the rotation phase within a knot of the handbook-prescribed $V_{R}$ value (for the conditions of this first flight) of 107 KIAS. Liftoff occurs approximately $3 \mathrm{~s}$ later (during which time the aircraft covers around $150 \mathrm{~m}$ with the main landing gear on the tarmac and the nose gear in the air) and the turboprop is airborne as it passes taxiway Bravo 1. Overall, with a weight of $6688 \mathrm{~kg}$ and operating at a density altitude of $-96 \mathrm{ft}$, the takeoff requires, on this occasion, around $53 \%$ of the available distance (TODA) and around $44 \%$ of the length of tarmac designated as available for the ground roll (TORA).

\section{F. Flight Testing Lessons Learned}

Although the flights conducted here did not form part of a certification process, we followed good practice outlined in certification testing guides wherever appropriate. For example, our inclusion of GPS and inertial data to augment the air data recorded in the course of each takeoff follows the guidance set out in Ref. [29]. A key takeaway here formed around the challenges illustrated, for example, in Fig. 6. Clearly, higher time-domain and spatial resolution systems are available today than those integrated in the test aircraft and used here, and we will aim to design future flight-test campaigns around much more up-to-date Global Navigation Satellite System and inertial referencing technology. Clearly, the integrated system is suitable for the vast majority of test cases flown by G-NFLA, but high-precision reconstruction of transient maneuvers (such as required here for, for example, identifying the exact point of liftoff) would benefit from recent augmentation technology (e.g., multiconstellation averaging, differential GPS), obviating the type of time-consuming modeling and snap-to-road methodology employed here. Indeed, even the sophisticated data fusion technology powering the attitude and positioning capability of a modern smartphone could provide a significant step-up in resolution and precision, given an adequate view of the sky (e.g., the GPS + GLONASS + Galileo + QZSS + BeiDou + magnetic compass suite featured by some currentgeneration smartphones).

The processing of the air data has also yielded useful take-home lessons. As discussed previously, this proved more laborious than expected and brought with it data quality issues. The precise and rigorously validated ground-based wind measurements of the airport served as useful ground truth, but at a low time resolution and in a single location. We augmented this, as described previously, with the own air data of the aircraft, which, for most flight testing purposes, offer excellent accuracy and high resolution. However, we once again ran into the issue of transient phenomena in the case of the surgical deconstruction of the ground roll. The sensitivity of the air data to abrupt changes in power setting (e.g., ground-roll start) limits the usability of the air data for the estimation of TAS (defined in relation to the unaffected air mass), as the local flow speed over the pitot-static system becomes significantly different from the TAS due to the proximity of the propellers (ideal for operational reasons, less so for the niche application of highresolution ground-roll reconstruction). The ideal solution may be to perform such tests on low-level wind-shear alert systemequipped runways or, if unavailable, setting up an ad hoc network of sensors to provide a similar capability (see Ref. [30] for a further discussion of these challenges associated with the high-resolution reconstruction of ground rolls)

\section{Validation}

\section{A. Monte Carlo Setup}

Let us now consider the Monte Carlo simulation setup that we pressed into the service of validating the framework sketched out in Fig. 2. We have selected seven probabilistic inputs for this exercise, divided into three categories: propulsion, aerodynamics, and piloting technique (see Table 2).

Following a very precise reconstruction of the performance of the TPE331-10UR-513H turboprop engines, the key sources of remaining propulsion uncertainty are the integration losses; we use Kundu's [22] estimates here (first line of Table 2). The parameters of the distributions of aerodynamic parameters fed into the simulations are based on the data presented in Sec. IV.C, assuming normal 


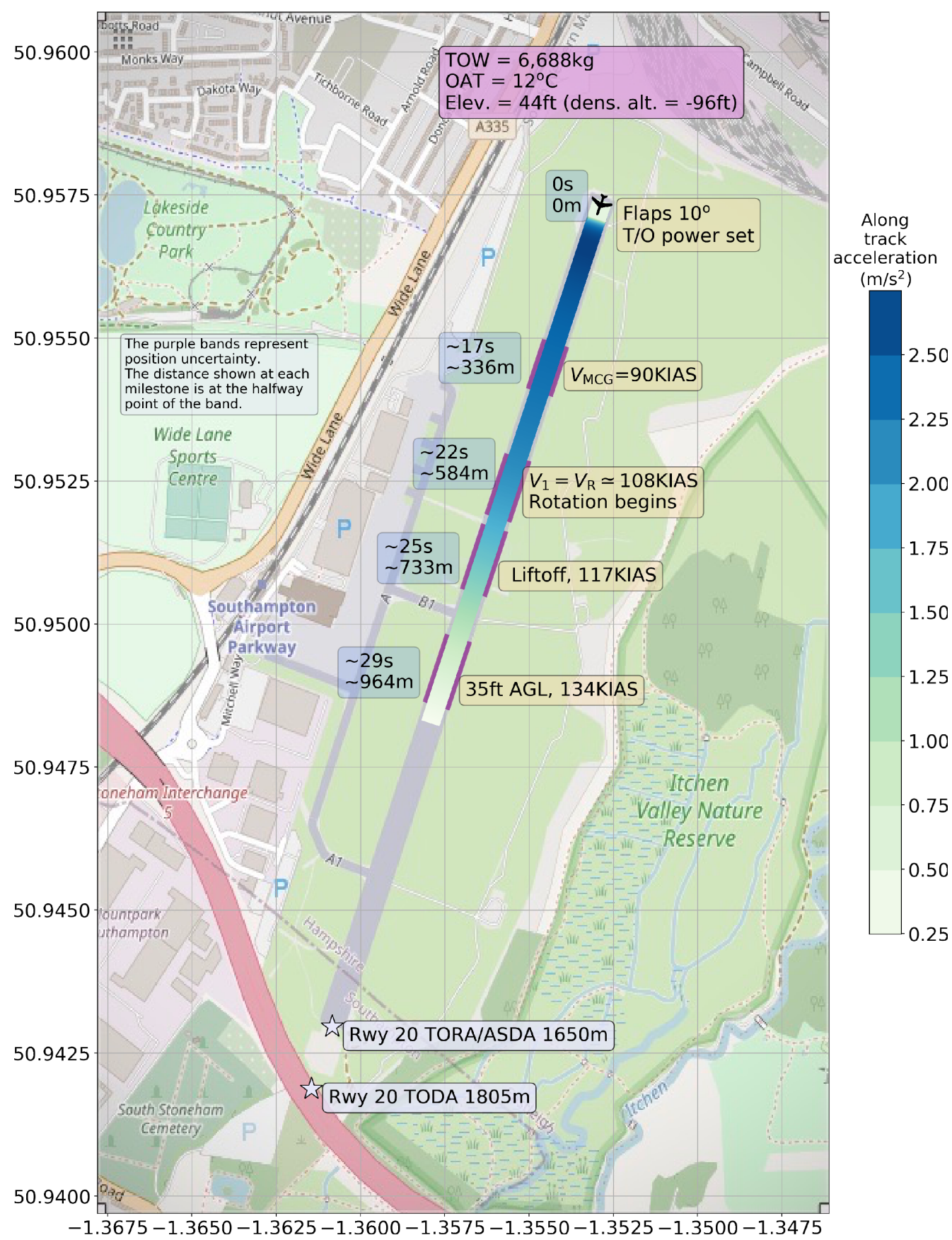

Fig. 9 Reconstruction of a takeoff from test flight data (flight 1) (TOW = take-off weight; AGL = above ground level; Elev = elevation; dens. alt. = density altitude; Rwy = runway).

Table 2 Monte Carlo inputs for the model runs used in the validation study

\begin{tabular}{lccc}
\hline \hline Input & Distribution & Mean & Standard deviation \\
\hline \multicolumn{4}{c}{ Propulsion } \\
\hline Engine installation factor & Normal & 0.91 & 0.005 \\
\hline \multicolumn{4}{c}{ Aerodynamics } \\
\hline Lift curve slope $a_{1}^{\text {TO }}$ & Normal & $5.8 \mathrm{rad}^{-1}$ & $0.05 \mathrm{rad}^{-1}$ \\
Lift curve intercept $C_{L 0}^{\mathrm{TO}}$ & Normal & 0.523 & 0.004 \\
Induced drag coefficient $K$ & Normal & 0.056 & 0.0005 \\
Zero-lift drag coefficient $C_{D 0}^{\text {TO }}$ & Normal & 0.072 & 0.0005 \\
\hline \multicolumn{4}{c}{ Piloting technique } \\
\hline Maximum angle of rotation & Normal & $\approx 3 \mathrm{deg}$ \\
Rotation rate & Normal & $\approx 3 \mathrm{deg} / \mathrm{s}$ & $0.5 \mathrm{deg} / \mathrm{s}$ \\
\hline \hline
\end{tabular}

All parameters relate to the takeoff configuration. distributions. Finally, we assume a sigmoidal rotation input for the rotation of the airplane, starting from no elevator deflection to a deflection that results in a target maximum rotation angle (first line of the "piloting technique" section of Table 2 ), with a rate of transition between the two as specified in the last row of the table. The distribution mean figures shown here are representative values; the exact value for each simulation was that captured from the rate and maximum angle of rotation of the test flight being matched.

All other parameters of each simulation (not listed here) are assumed constant for each member of their respective Monte Carlo ensembles; for example, the takeoff weight of the aircraft on each validation flight was known very precisely, and so there was no need for a probabilistic treatment.

The choice of Monte Carlo input values is consistent with the philosophy that we are aiming to validate the structure of the model and its implementation (rather than its inputs), and so the goal is to have inputs that are as close to the observed reality as possible. 
The data reported on in the next section are the results of feeding the aforementioned distributions (and the appropriate single values for other parameters) into the framework depicted in Fig. 2; eight Monte Carlo ensembles were generated, one for each validation flight.

\section{B. Validation Results}

Let us now match the flight-test data for the eight flight tests against the corresponding simulation results generated via the Monte Carlo setup presented previously.

At the beginning of this paper, we set out two requirements, around which we designed the simulation capability described in Sec. III: that it needs to be fast enough to plausibly serve as a real-time decision support tool running on a laptop in a design meeting or in a preliminary discussion with a customer, and that it must have its accuracy benchmarked against flight tests.

The first of these requirements is easily tested. The author's $3.1 \mathrm{GHz}$ Intel Core i5-powered laptop can comfortably run an iteration of the framework depicted in Fig. 2 in just under two-tenths of a second (simulating around 40 "real" seconds of flight). With Fig. 3 as a guide, this can be translated into around half a minute for getting to within $10 \mathrm{~m}$ or so of the mean and the standard deviation of the takeoff distance distribution, sufficient for the purposes of supporting a design discussion. If more accuracy is desired, such as we did for the validation experiments described here, one may continue to about $20 \mathrm{~min}$ to converge the statistical moments to within a meter or so (or around $0.1 \%$ of the takeoff distance). With the Monte Carlo method offering linear speedups, multicore systems

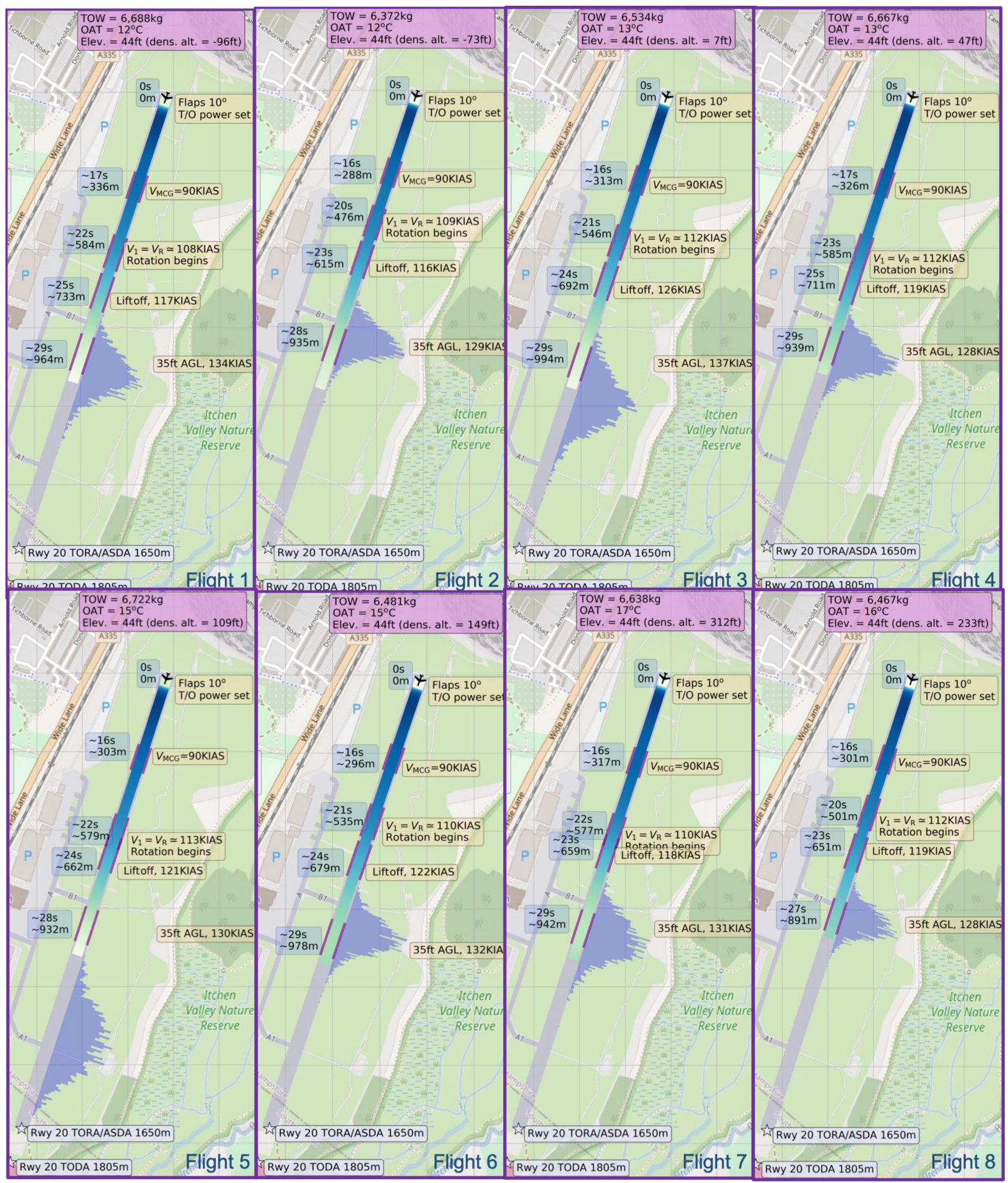

Fig. 10 Takeoff distance (to $35 \mathrm{ft}$ screen height) Monte Carlo histograms in blue, shown against the corresponding flight-test data. 
allow the division of these figures by the number of available cores; moreover, significant further speedups (such as may be required for a real-time onboard system) could be achieved by implementing the methodology in, say, $\mathrm{C}++$.

Let us now address the second part of the question by comparing the eight validation flights against simulation setup such that they match the conditions of the test flights as closely as possible. Consider the results depicted in Fig. 10, where each tile depicts one of the eight takeoffs and the distribution of takeoff distances (35 ft screen height reached) generated via 2000 iterations of the Monte Carlo simulation of that takeoff. The resulting histogram (in blue) of takeoff distances is aligned with the appropriate location along the runway. The purple bands either side of the runway, which can be seen underneath (or, geographically, to the west-north-west of the histogram) delineate the area within which we estimate that each test takeoff ended, that is, where G-NFLA passed $35 \mathrm{ft}$ above ground level.

The simulations used the takeoff mass calculated from the load sheets of each flight, and our best reconstruction of the environmental conditions that prevailed at the time of the takeoff, as laid out in Sec. IV, with a normally distributed wind perturbation of a $2 \mathrm{kt}$ standard deviation. Further, we used a set of sigmoid functions to approximate the spool-up profile of the engine deck and the pitch-up control input leading to the rotation of the aircraft. In terms of the aerodynamics and propulsion performance of the aircraft, as well as the corresponding uncertainty margins, we used the model outlined in Sec. IV.B and IV.C.

The first conclusion is the relatively large spread of the predicted takeoff distances, which highlights the way in which even relatively narrow uncertainties (such as those we specified here on the aerodynamic coefficients, the propulsion installation factor, and pilot inputs) can build up over the $30 \mathrm{~s} / \mathrm{a}$ kilometer or so it takes the Jetstream to clear $35 \mathrm{ft}$ from a standing start; the farthest outliers typically exceed the mean by over $20 \%$.

The second observation one might make is that the purple band representing the actual takeoff distance range of each test flight generally aligns well with the predicted distribution of distances; this is true of seven of the eight cases, with the exception being flight 5 . The probable cause of this anomaly is that, during the takeoff run, there was a deviation from the METAR-reported wind in direction and/or speed.

This hypothesis is supported by a comparison between flights 5 and 6 . Flight 5 features the heaviest takeoff weight of the campaign, whereas on flight 6 , G-NFLA weighed less by $241 \mathrm{~kg}$. The "baseline" wind speeds recorded in the METARs (see Table 1) were very similar at the time of the two flights (14 and 15-16 kt, respectively, from 190 to $200 \mathrm{deg})$, as was the OAT $\left(15^{\circ} \mathrm{C}\right.$ in both cases). Yet, the center of the " $35 \mathrm{ft}$ screen height reached" uncertainty band was at the $932 \mathrm{~m}$ mark on flight 5, whereas flight 6 (lighter by the weight of three to four adults) took $46 \mathrm{~m}$ longer to reach the equivalent milestone. With

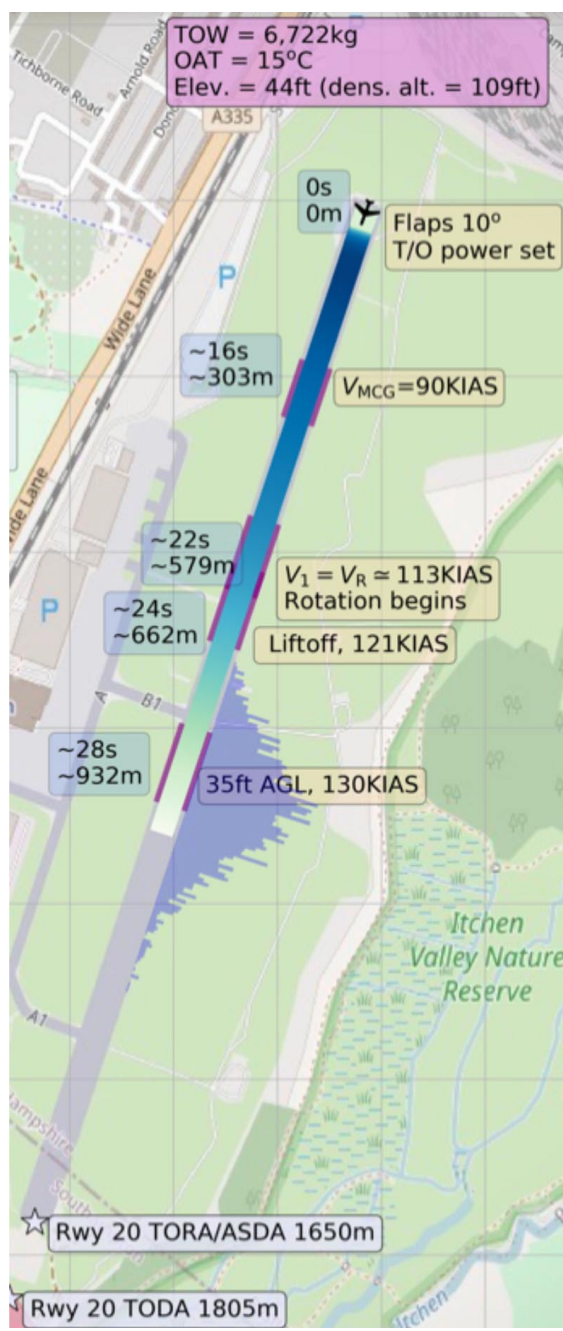

Fig. 12 Flight 5 simulation results with a headwind gust introduced at $15 \mathrm{~s}$ into the ground roll.

little evidence of significant inconsistencies in piloting technique from one flight to the next, the strongest possibility remains a change in the runway component of the wind speed (with the METAR indicating directional variability between 170 and $240 \mathrm{deg}$ ) or a wind gust during the takeoff run.

The air data recorded by the aircraft offer further evidence in support of the wind variability hypothesis. Although, as discussed

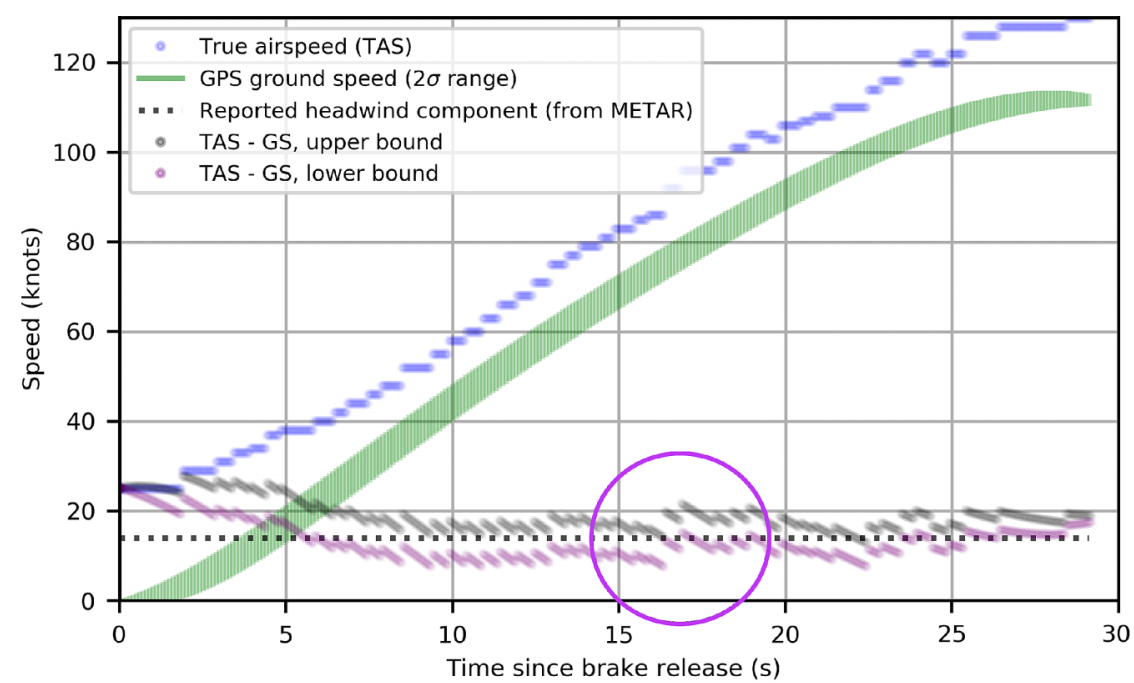

Fig. 11 Air data recorded by G-NFLA on flight 5; the circle highlights the location of a sudden change in the headwind component. 
in Sec. IV, propulsion effects render the TAS minus ground speed signal unsuitable for reconstructing actual headwind values, abrupt variations in it do offer an indication of changes in wind speed and direction. Examining the air data traces for flight 5 does indeed reveal evidence of such an event, featuring a clear jump in the estimated headwind (circled in Fig. 11).

To test the wind variation hypothesis (as a possible reason for the flight 5 anomaly) directly, we repeated the flight 5 simulations, this time injecting a gust into the simulation, just after the $15 \mathrm{~s}$ mark (the location of the discontinuity highlighted in Fig. 11), temporarily increasing the headwind to $30 \mathrm{kt}$ (the value seen in the METAR corresponding to the previous flight). The results of this fresh simulation are shown via the histogram in Fig. 12, revealing a good alignment between the distribution of simulated takeoffs and the "35 ft screen height cleared" zone derived from the data recorded on flight 5 , in keeping with the results of the other seven flights.

\section{Conclusions}

The study described in this paper set out to propose a Monte Carlo model that allows the rapid, probabilistic assessment of the takeoff performance of an aircraft (anywhere between the earliest stages of its design and in service) and assess its accuracy against real-life data acquired as part of a flight-test campaign. Along the way, the research effort reported on here also turned into an exercise in understanding the challenges and pitfalls of the reconstruction of takeoff flight-test data in a way that quantifies its uncertainties. This was made especially challenging by the limitations of the LTN-90, a state-of-the-art device in its time, but now four decades behind modern GPS and IRS technology.

The analysis of the air data also provided some interesting challenges. Although G-NFLA features much useful instrumentation and logging capability beyond what production variants of the Jetstream 3100 are equipped with, they offer (not entirely unreasonably) far more to the flight-test engineer when the aircraft is airborne; the peculiarities of takeoff (large speed and power setting transients, etc.) are on the margins of their capabilities. One example is the AOA vane, the signal of which is unreliable at the low speeds typical of much of the takeoff.

Let this paper conclude by summarizing the findings, results, and recommendations of the work reported previously:

1) It is hoped that readers aiming to conduct their own computational model vs flight-test data validation study will find the analysis a useful template for a comparison with quantified uncertainties and will be able to design a more effective experiment avoiding some of the pitfalls that were encountered along the way.

2) As for the model itself, it has been demonstrated that its execution speed and accuracy make it a useful prediction tool in a range of settings. The main target use case is its ability to support design discussions in the early conceptual phase or perhaps even at the time when the design brief is drawn up (the method being sufficiently fast to support discussions with real-time "what ifs"), or the initial feasibility of a tentative design is tested. Finely poised concepts in the emerging urban mobility sector may benefit particularly from early quantification of takeoff performance uncertainty; the early generation of probabilistic noise footprint maps may be another particularly interesting application candidate.

3) The validation study presented here provides strong evidence for the thesis that very efficient, lightweight point mass models can offer a high level of accuracy within a Monte Carlo framework, within the requirements of design studies and most operational applications as well.

4) It was found that solving the governing equations via an adaptive step-size approach (using the Bogacki-Shampine formula) is ideally suited for dealing with the transients associated with takeoff, as it speeds up the solution process significantly, while performing robustly and in a stable manner.

5) Much takeoff modeling at the conceptual design stage typically relies on analytical models at present. They are fast to compute, but their rigidity (inability to model contingencies, different piloting techniques, detailed weather and runway geography, etc.) limits their usefulness. Conversely, full-flight simulators based on blade element models or higher-fidelity simulation technology is not sufficiently fast for practical Monte Carlo studies, an essential step in understanding the downstream impact of uncertainties. In this paper, it was shown that a point mass model engineered with modularity and flexibility in mind offers the flexibility of the latter at a fraction of its computational cost. (Note that this claim is made only for takeoff performance modeling. A detailed dynamics model with a complete set of inertial properties and derivatives may be required for other maneuvers.)

6) The study presented here is also an argument in favor of the suitability of the point mass model described for precise and rapid operational field performance prediction in probabilistic terms, potentially offering more operational flexibility than conventional, highly conservative, rigid, "handbook-based" methods. A typical use case might be a tilt-rotor-type fixed-wing drone operating out of varied, constrained environments (e.g., ship based) offering a variety of takeoff techniques depending on payload and environmental and geographical conditions. The approach detailed here may be used to generate an optimal thrust vectoring or high-lift system deployment schedule, or compute a payload limit based on the rapid assessment of a large number of possible propulsion system failure scenarios adapted to the exact environment being considered.

7) The probabilistic nature of the model allows it to be used to facilitate a quantified risk-based approach to field performance, especially useful in cases where the impact of failure can only be damage to a low-cost vehicle.

8) The low computational cost of the model, combined with the increasing speed of graphical processing units, could yield a powerful, real-time, onboard anomaly detection tool that could make hundreds of probabilistically based "go/no-go" decisions in the course of the ground roll of a vehicle, by constantly assessing recomputed predicted performance vs TORA.

To maximize the benefit to the community of the model described here, it will form part of the Aircraft Design Recipes in Python (see https://github.com/sobester/ADRpy) open-source library.

\section{Acknowledgments}

The flights were funded by the School of Engineering of the University of Southampton as part of an annual educational test campaign integrated into the aeronautics and astronautics undergraduate program of the school. The development and validation of the takeoff performance model were funded by the UK Engineering and Physical Sciences Research Council under grant number EP/R009953/1 (CASCADE project). The author would like to thank Tim Takahashi, Andy Keane, and Jim Scanlan for useful discussions on uncertainty quantification, takeoff performance, and first-order performance modeling. Special thanks also go to the National Flying Laboratory Centre team from Cranfield University, who not only operated the flights, but also patiently answered the author's questions afterward (in particular, Nick Lawson and Alastair Cooke).

\section{References}

[1] Zollitsch, A. W., Mumm, N. C., Holzapfel, F., and Schumann, J., "System Health Management for Safe Automatic Take-Off," AIAA Paper 2019-1960, Jan. 2019, pp. 1-14. https://doi.org/10.2514/6.2019-1960

[2] Anon., Global Climatic Data for Developing Military Products (MILHDBK-310), Dept. of Defense, Washington, D.C., 1997, pp. 1-123.

[3] Allaire, D., Noel, G., Willcox, K., and Cointin, R., "Uncertainty Quantification of an Aviation Environmental Toolsuite," Reliability Engineering \& System Safety, Vol. 126, June 2014, pp. 14-24. https://doi.org/10.1016/j.ress.2014.01.002

[4] Wendorff, A., Alonso, J. J., Bieniawski, S. R., and Whitehead, B. T., "Integrating Aerodynamic Uncertainty into Aircraft Maneuvers During Conceptual Design,” AIAA Paper 2016-3999, June 2016, pp. 1-17. https://doi.org/10.2514/6.2016-3999

[5] Wendorff, A., Alonso, J. J., and Bieniawski, S. R., "Using Multiple Information Sources to Construct Stochastic Databases to Quantify Uncertainty in Certification Maneuvers," AIAA Paper 2016-1420, 
Jan. 2016, pp. 1-16. https://doi.org/10.2514/6.2016-1420

[6] Ng, L. W. T., and Willcox, K. E., "Monte Carlo Information-Reuse Approach to Aircraft Conceptual Design Optimization Under Uncertainty," Journal of Aircraft, Vol. 53, No. 2, 2016, pp. 427-438. https://doi.org/10.2514/1.C033352

[7] Takahashi, T. T., Wood, D. L., and Bays, L. V., "An Introduction to the Impact of Pilot Techniques upon "Certified" Field Performance," AIAA Paper 2017-0007, Jan. 2017, pp. 1-33. https://doi.org/10.2514/6.2017-0007

[8] Takahashi, T. T., Wood, D. L., and Bays, L. V., "The Effect of Aerodynamic and Propulsive Uncertainty upon Certified Takeoff Performance," AIAA Paper 2017-3420, June 2017, pp. 1-22. https://doi.org/10.2514/6.2017-3420

[9] Bays, L. V., and Halpin, K. E., "Improved Safety and Capability via Direct Computation of Takeoff and Landing Performance Data," AIAA Paper 2014-2154, June 2014, pp. 1-42. https://doi.org/10.2514/6.2014-2154

[10] Airbus Customer Services, "Getting to Grips with Aircraft Performance," Airbus Customer Services Flight Operations Support and Line Assistance Manual, Jan. 2002.

[11] Moore, K. R., and Ning, A., "Takeoff and Performance Trade-Offs of Retrofit Distributed Electric Propulsion for Urban Transport," Journal of Aircraft, Vol. 56, No. 5, 2019, pp. 1880-1892. https://doi.org/10.2514/1.C035321

[12] Blake, W., "Jet Transport Performance Methods," Boeing Commercial Airplanes TR D6-1420, Seattle, WA, March 2009.

[13] Takahashi, T., Aircraft Performance and Sizing, Momentum Press, New York, 2017, Chap. 6.

[14] Bogacki, P., and Shampine, L. F., "A 3(2) Pair of Runge-Kutta Formulas," Applied Mathematics Letters, Vol. 2, No. 4, 1989, pp. 321-325. https://doi.org/10.1016/0893-9659(89)90079-7

[15] Virtanen, P., Gommers, R., Oliphant, T. E., Haberland, M., Reddy, T., Cournapeau, D., Burovski, E., Peterson, P., Weckesser, W., Bright, J., and van der Walt, S. J., "SciPy 1.0: Fundamental Algorithms for Scientific Computing in Python," Nature Methods, Vol. 17, No. 3, 2020, pp. 261-272. https://doi.org/10.1038/s41592-019-0686-2

[16] Siva, C., Murugan, M. S., and Ganguli, R., "Uncertainty Quantification in Helicopter Performance Using Monte Carlo Simulations," Journal of Aircraft, Vol. 48, No. 5, 2011, pp. 1503-1511. https://doi.org/10.2514/1.C000288

[17] Khurana, M. S., Russell, C. R., and Scott, R., "Uncertainty Quantification of a Rotorcraft Conceptual Sizing Toolsuite," AIAA Paper
2019-1728, Jan. 2019, pp. 1-24.

https://doi.org/10.2514/6.2019-1728

[18] Sóbester, A., Czerski, H., Zapponi, N., and Castro, I., "High-Altitude Gas Balloon Trajectory Prediction: A Monte Carlo Model," AIAA Journal, Vol. 52, No. 4, 2014, pp. 832-842. https://doi.org/10.2514/1.J052900

[19] Wood, D. L., Takahashi, T. T., and Bays, L. V., "Experimental Investigation of Typical Aircraft Field Performance Versus Predicted Performance Targets," AIAA Paper 2017-3276, June 2017, pp. 1-18. https://doi.org/10.2514/6.2017-3276

[20] Anon., "Type Certificate Data Sheet E4WE," Dept. of Transportation, Federal Aviation Administration, 2014.

[21] Anon., "TPE331-10 Turboprop Engine," Honeywell International Inc., 2006, https://aerospace.honeywell.com/content/dam/aerobt/en/docum ents/learn/products/engines/brochures/N61-1491-000-000-TPE331-10 TurbopropEngine-bro.pdf.

[22] Kundu, A. K., Aircraft Design, Cambridge Aerospace Series, Cambridge Univ. Press, Cambridge, England, U.K., 2010, Chap. 10. https://doi.org/10.1017/CBO9780511844652

[23] Anon., "Jetstream Series 3100 Crew Manual, Volume 1," Prestwick International Airport SA.4-3100/CM/08C, Ayrshire, Scotland, 2002.

[24] Cooke, A. K., "Simulation Model of the NFLC Jetstream 31," Cranfield Univ., College of Aeronautics Rept. 0402, Cranfield, U.K., 2006.

[25] Lawson, N. J., Jacques, H., Gautrey, J. E., Cooke, A. K., Holt, J. C., and Garry, K. P., "Jetstream 31 National Flying Laboratory: Lift and Drag Measurement and Modelling," Aerospace Science and Technology, Vol. 60, Jan. 2017, pp. 84-95. https://doi.org/10.1016/j.ast.2016.11.001

[26] Bennett, C. J., Lawson, N. J., Gautrey, J. E., and Cooke, A., "CFD Simulation of Flow Around Angle of Attack and Sideslip Angle Vanes on a BAe Jetstream 3102-Part 1," Aerospace Science and Technology, Vol. 68, Sept. 2017, pp. 561-576. https://doi.org/10.1016/j.ast.2017.03.015

[27] McCormick, B., Aerodynamics, Aeronautics, and Flight Mechanics, Wiley, New York, 1994, Chap. 3.

[28] Gudmundsson, S., General Aviation Aircraft Design: Applied Methods and Procedures, Elsevier Science, Oxford, U.K., 2013, Chap. 9.

[29] Anon., "Flight Test Guide for Certification of Part 23 airplanes AC238C," U.S. Dept. of Transportation, Federal Aviation Administration, Advisory Circular, 2010.

[30] Anon., "Runway Side Excursion During Attempted Takeoff in Strong and Gusty Crosswind Conditions Continental Airlines Flight 1404 Boeing 737-500, N18611 Denver, Colorado December 20, 2008," Aircraft Accident Rept. National Transportation Safety Board AAR-10/04, PB2010-910404, 2010. 